\title{
ON PERIODIC SOLUTIONS FOR FIRST-ORDER DIFFERENTIAL EQUATIONS INVOLVING THE DISTRIBUTIONAL HENSTOCK-KURZWEIL INTEGRAL
}

\author{
WEI LIU ${ }^{凶}$, GUOJU YE, YING WANG and XUEYUAN ZHOU
}

(Received 27 October 2011)

\begin{abstract}
The purpose of this paper is to study the existence of periodic solutions and the topological structure of the solution set of first-order differential equations involving the distributional Henstock-Kurzweil integral. The distributional Henstock-Kurzweil integral is a general integral, which includes the Lebesgue and Henstock-Kurzweil integrals. The main results extend some previously known results in the literature.
\end{abstract}

2010 Mathematics subject classification: primary 26A39; secondary 28B05, 39A23, 46F05, $46 \mathrm{G} 12$.

Keywords and phrases: periodic boundary value problem, distributional Henstock-Kurzweil integral, distributional derivative, extremal solutions, fixed point.

\section{Introduction}

In this paper, we consider the periodic boundary value problem (PBVP) for the firstorder differential equation

$$
D x=f(t, x), \quad x(0)=x(T),
$$

where $D x$ stands for the distributional derivative of $x \in C([0, T]), 0<T<\infty$, and $f$ is a distribution (generalised function).

Distributions and their derivatives are defined at the beginning of Section 2. The notion of a distributional derivative is very general, including, for example, ordinary derivatives and approximate derivatives. The first-order PBVP for ordinary differential equations

$$
\frac{d x}{d t}=f(t, x), \quad x(0)=x(T),
$$

with ordinary derivative $d x / d t$ and $f:[0, T] \times \mathbb{R}^{n} \rightarrow \mathbb{R}^{n}$ has been studied extensively in recent years; see, for example, $[4,5,10,11,16]$. However, as far as we know, few

Supported by NNSF of China (10871059) and the Fundamental Research Funds for the Central Universities.

(c) 2012 Australian Mathematical Publishing Association Inc. 0004-9727/2012 \$16.00 
papers have applied distributional derivatives to study PBVP. In this paper, by using distributional derivatives, we study the PBVP (1.1) and obtain some interesting results.

This paper is organised as follows. In Section 2 we introduce a general integral called the distributional Henstock-Kurzweil integral or $D_{H K}$-integral. We say that a distribution $f$ is $D_{H K}$-integrable on $[a, b] \subset \mathbb{R}$ if there is a unique continuous function $F$ on $[a, b]$ with $F(a)=0$ whose distributional derivative is $f$. From the definition of the $D_{H K}$-integral, we know that the $D_{H K}$-integral includes the Riemann, Lebesgue, Henstock-Kurzweil $(H K)$ and wide Denjoy integrals (for details, see [2, 6, 7, 14, 15]). The space of integrable distributions is a Banach space and has many good properties; see $[8,9]$.

In Section 3, with the $D_{H K}$-integral and the distributional derivative, we generalise the PBVP (1.2) to (1.1) and apply the well-known Schauder fixed point theorem to study the existence of multiple solutions of (1.1). More precisely, by using the Vidossich theorem, we show that the solution set is an $R_{\delta}$, that is, it is homeomorphic to the intersection of a decreasing sequence of compact absolute retracts. Results in this paper are generalisations of corresponding results in the references.

\section{The distributional Henstock-Kurzweil integral}

In this section, we present the definition and some basic properties of the distributional Henstock-Kurzweil integral.

Define the space

$$
C_{c}^{\infty}=\left\{\phi: \mathbb{R} \rightarrow \mathbb{R} \mid \phi \in C^{\infty} \text { and } \phi \text { has compact support in } \mathbb{R}\right\},
$$

where the support of a function $\phi$ is the closure of the set on which $\phi$ does not vanish, denoted by $\operatorname{supp}(\phi)$. A sequence $\left\{\phi_{n}\right\} \subset C_{c}^{\infty}$ converges to $\phi \in C_{c}^{\infty}$ if there is a compact set $K$ such that all $\phi_{n}$ have supports in $K$ and, for every $m \in \mathbb{N}$, the sequence of derivatives $\phi_{n}^{(m)}$ converges to $\phi^{(m)}$ uniformly on $K$. Denote $C_{c}^{\infty}$ endowed with this convergence property by $\mathcal{D}$. Also, $\phi$ is called a test function if $\phi \in \mathcal{D}$. Distributions are defined to be continuous linear functionals on $\mathcal{D}$. The space of distributions is denoted by $\mathcal{D}^{\prime}$, which is the dual space of $\mathcal{D}$. That is, if $f \in \mathcal{D}^{\prime}$ then $f: \mathcal{D} \rightarrow \mathbb{R}$, and we write $\langle f, \phi\rangle \in \mathbb{R}$, for $\phi \in \mathcal{D}$.

For all $f \in \mathcal{D}^{\prime}$, we define the distributional derivative $D f$ of $f$ to be a distribution satisfying $\langle D f, \phi\rangle=-\left\langle f, \phi^{\prime}\right\rangle$, where $\phi$ is a test function. With this definition, all distributions have derivatives of all orders and each derivative is a distribution.

Let $(a, b)$ be an open interval in $\mathbb{R}$. We define

$$
\mathcal{D}((a, b))=\left\{\phi:(a, b) \rightarrow \mathbb{R} \mid \phi \in C_{c}^{\infty} \text { and } \phi \text { has compact support in }(a, b)\right\} .
$$

The dual space of $\mathcal{D}((a, b))$ is denoted by $\mathcal{D}^{\prime}((a, b))$.

Define $C([a, b])$ to be the space of continuous functions on $[a, b]$, and

$$
B_{C}=\{F \in C([a, b]) \mid F(a)=0\} .
$$

Note that $B_{C}$ is a Banach space with the uniform norm $\|F\|_{\infty}=\max _{[a, b]}|F|$. 
We are now able to introduce the definition of the $D_{H K}$-integral.

Definition 2.1. A distribution $f$ is distributionally Henstock-Kurzweil integrable (or $D_{H K}$-integrable) on $[a, b]$ if $f$ is the distributional derivative of a continuous function $F \in B_{C}$.

The space of $D_{H K}$-integrable distributions is defined by

$$
D_{H K}=\left\{f \in \mathcal{D}^{\prime}((a, b)) \mid f=D F \text { for some } F \in B_{C}\right\} .
$$

With this definition, if $f \in D_{H K}$ then, for all $\phi \in \mathcal{D}((a, b))$,

$$
\langle f, \phi\rangle=\langle D F, \phi\rangle=-\left\langle F, \phi^{\prime}\right\rangle=-\int_{a}^{b} F \phi^{\prime} .
$$

The $D_{H K}$-integral of $f$ on $[a, b]$ is given by $\left(D_{H K}\right) \int_{a}^{b} f=F(b)$, where $F$ is called the primitive of $f$ and ' $\left(D_{H K}\right) \int$ ' denotes the $D_{H K}$-integral. As usual, if a function $F \in C([a, b])$ and $\left(D_{H K}\right) \int_{a}^{b} f=F(b)-F(a)$, then the function $F$ is a primitive of $f$. Notice that if $f \in D_{H K}$ then $f$ has many primitives in $C([a, b])$, all differing by a constant, but $f$ has exactly one primitive in $B_{C}$.

REMARK 2.2. Integrals defined in the same way have also been proposed in other papers. For example, Ang et al. [1] defined an integral in the plane and called it the $G$-integral, and Talvila [15] defined the $A_{C}$-integral on the extended real line. In fact, these two integrals are equivalent to the $D_{H K}$-integral for one-dimensional intervals.

The following result is known as the fundamental theorem of calculus.

LEMMA 2.3 [15, Theorem 4].

(a) Let $f \in D_{H K}$, and define $F(t)=\left(D_{H K}\right) \int_{a}^{t} f$. Then $F \in B_{C}$ and $D F=f$.

(b) Let $F \in C([a, b])$. Then $\left(D_{H K}\right) \int_{a}^{t} D F=F(t)-F(a)$ for all $t \in[a, b]$.

EXAMPLE 2.4. We know that the primitive function $F$ of the $H K$-integrable function $f$ is $A C G^{*}$ (generalised absolutely continuous; see [6, 14]). In [6, Example 6.6], Lee pointed out that if $F$ is a continuous function and pointwise differentiable nearly everywhere on $[a, b]$, that is, everywhere except perhaps for a countable number of points in $[a, b]$, then $F$ is $A C G^{*}$. Furthermore, if $F$ is a continuous function which is differentiable nowhere on $[a, b]$, then $F$ is not $A C G^{*}$. Therefore, if $F \in C([a, b])$ but is differentiable nowhere on $[a, b]$, then $D F$ exists and is $D_{H K}$-integrable but not $H K$-integrable. Conversely, if $F$ is $A C G^{*}$ then it also belongs to $C([a, b])$. Therefore, $F^{\prime}$ is not only $H K$-integrable but also $D_{H K}$-integrable. Here, $F^{\prime}$ denotes the ordinary derivative of $F$.

This example shows that the $D_{H K}$-integral includes the $H K$-integral, and hence the Lebesgue and Riemann integrals. 
We now give some other results about the distributional derivative and the $D_{H K^{-}}$ integral.

Let $F, G$ be two functions, and denote by $F \circ G$ the composition of $F$ and $G$.

Definition 2.5 [15, Definition 10, Derivative of composition of continuous functions]. Let $F, G \in C([a, b])$. Then $(D F \circ G) D G:=D(F \circ G)$, that is, for all $\phi \in \mathcal{D}((a, b))$,

$$
\langle(D F \circ G) D G, \phi\rangle=\langle D(F \circ G), \phi\rangle=-\left\langle F \circ G, \phi^{\prime}\right\rangle=-\int_{a}^{b}(F \circ G)(t) \phi^{\prime}(t) d t .
$$

Lemma $2.6\left[15\right.$, Theorem 11]. Suppose that $f \in D_{H K}$ and $D F=f$, where $F \in$ $C([\alpha, \beta]),-\infty<\alpha<\beta<+\infty$. Let $\alpha<a<b<\beta$. If $G \in C([a, b])$ then

$$
\int_{G(a)}^{G(b)} f=\int_{a}^{b}(f \circ G) D G=(F \circ G)(b)-(F \circ G)(a) .
$$

If $G \in C((a, b))$ and $\lim _{t \rightarrow a^{+}} G(t)=\alpha$ and $\lim _{t \rightarrow b^{-}} G(t)=\beta$ then

$$
\int_{\alpha}^{\beta} f=\int_{a}^{b}(f \circ G) D G=F(\beta)-F(\alpha) .
$$

Lemma 2.7. Let $f, g$ be the distributional derivatives of $F, G$, respectively, where $F, G \in C([a, b])$. Then

$$
D(F G)=f G+F g .
$$

Proof. It follows from the definition of distributional derivative and (2.1) that

$$
\begin{aligned}
\langle D(F G), \phi\rangle & =-\left\langle F G, \phi^{\prime}\right\rangle=-\int_{a}^{b} F\left(G \phi^{\prime}\right) \\
& =-\int_{a}^{b} F(D(G \phi)-g \phi)=-\int_{a}^{b} F D(G \phi)+\int_{a}^{b} F g \phi \\
& =\int_{a}^{b} f G \phi+\int_{a}^{b} F g \phi=\int_{a}^{b}(F g+f G) \phi \\
& =\langle F g+f G, \phi\rangle .
\end{aligned}
$$

Consequently, the result holds.

If $g:[a, b] \rightarrow \mathbb{R}$, its variation is $V g=\sup \sum_{n}\left|g\left(t_{n}\right)-g\left(s_{n}\right)\right|$, where the supremum is taken over every sequence $\left\{\left(t_{n}, s_{n}\right)\right\}$ of disjoint intervals in $[a, b]$. If $V g<\infty$ then $g$ is called a function with bounded variation. Denote the set of functions with bounded variation by $\mathcal{B V}$. As it is known that the dual space of $D_{H K}$ is $\mathcal{B V}$ (see details in [15]), we have the next result.

Lemma 2.8 [15, Definition 6, Integration by parts]. Let $f \in D_{H K}$ and $g \in \mathcal{B V}$. Define $f g=D H$, where $H(t)=F(t) g(t)-\int_{a}^{t} F d g$. Then $f g \in D_{H K}$ and

$$
\int_{a}^{b} f g=F(b) g(b)-\int_{a}^{b} F d g .
$$


For $f \in D_{H K}$ and $F \in B_{C}$ with $D F=f$, we define the Alexiewicz norm by

$$
\|f\|=\|F\|_{\infty}=\max _{[a, b]}|F| .
$$

The following result has been proved.

Lemma 2.9 [15, Theorem 2]. With the Alexiewicz norm, $D_{H K}$ is a Banach space.

It is known that there is a pointwise ordering in $C([a, b])$ (so it is with $B_{C}$ ), that is, $u \leq v$ in $C([a, b])$ if and only if $u(t) \leq v(t)$ for every $t \in[a, b]$. We now impose a partial ordering on $D_{H K}$ : for $f, g \in D_{H K}$, we say that $f \geq g$ (or $g \leq f$ ) if and only if $f-g$ is a positive measure (see [12, Definition 1.18] for the definition of positive measure) on $[a, b]$. By this definition, if $f, g \in D_{H K}$ then

$$
\left(D_{H K}\right) \int_{a}^{b} f \geq\left(D_{H K}\right) \int_{a}^{b} g \quad \text { whenever } f \geq g .
$$

According to the definition of this ordering, we also have the following result.

Lemma 2.10 [1, Corollary 1]. If $f_{1}, f_{2}, f_{3} \in \mathcal{D}^{\prime}((a, b)), f_{1} \leq f_{2} \leq f_{3}$, and if $f_{1}$ and $f_{3}$ are $D_{H K}$-integrable, then $f_{2}$ is also $D_{H K}$-integrable.

We say that a sequence $\left\{f_{n}\right\} \subset D_{H K}$ converges strongly to $f \in D_{H K}$ if $\left\|f_{n}-f\right\| \rightarrow 0$ as $n \rightarrow \infty$. The following two convergence theorems hold.

Lemma 2.11 [1, Corollary 4, Monotone convergence theorem for the $D_{H K}$-integral]. Let $\left\{f_{n}\right\}_{n=0}^{\infty}$ be a sequence in $D_{H K}$ such that $f_{0} \leq f_{1} \leq \cdots \leq f_{n} \leq \cdots$, and that $\left(D_{H K}\right) \int_{a}^{b} f_{n} \rightarrow A$ as $n \rightarrow \infty$. Then $f_{n} \rightarrow f$ in $D_{H K}$ and $\left(D_{H K}\right) \int_{a}^{b} f=A$.

Lemma 2.12 [1, Corollary 5, Dominated convergence theorem for the $D_{H K}$-integral]. Let $\left\{f_{n}\right\}_{n=0}^{\infty}$ be a sequence in $D_{H K}$ such that $f_{n} \rightarrow f$ in $\mathcal{D}^{\prime}$. Suppose that there exist $f_{-}, f_{+} \in D_{H K}$ satisfying $f_{-} \leq f_{n} \leq f_{+}$, for all $n \in \mathbb{N}$. Then $f \in D_{H K}$ and $\lim _{n \rightarrow \infty}\left(D_{H K}\right) \int_{a}^{b} f_{n}=\left(D_{H K}\right) \int_{a}^{b} f$.

\section{Periodic boundary value problems}

In this section, we shall study the first-order PBVP

$$
D x=f(t, x), \quad x(0)=x(T),
$$

where $D x$ denotes the distributional derivative of $x \in C([0, T])$ and $f$ is a distribution. Throughout this section, we denote by $D_{H K}$ (respectively $H K, L$ ) the space of $D_{H K^{-}}$ (respectively, $H K$-, Lebesgue) integrable functions and by ' $(*) \int$ ' the $*$-integral.

We now impose some assumptions on the distribution $f$.

( $\left.\mathrm{D}_{1}\right) f(\cdot, x(\cdot))$ is $D_{H K}$-integrable for every fixed $x \in C([0, T])$.

( $\left.\mathrm{D}_{2}\right) f(t, \cdot)$ is continuous for all $t \in[0, T]$. 
$\left(\mathrm{D}_{3}\right)$ There exist $y, z \in C([0, T])$, with $y(0) \leq y(T)$ and $z(0) \geq z(T)$, such that

$$
D y \leq f(\cdot, x) \leq D z \text { for all } x \in[y, z],
$$

where $D y$ and $D z$ are the distributional derivatives of $y$ and $z$ respectively, and the ordering interval $[y, z]=\{x \in C([0, T]) \mid y(t) \leq x(t) \leq z(t)$ for all $t \in[0, T]\}$.

Before coming to the main results in this paper, we give a result following from Lemma 2.3-that is, the PBVP (1.1) can be converted to an integral equation.

Lemma 3.1. Let the distribution $f$ satisfy $\left(\mathrm{D}_{1}\right)-\left(\mathrm{D}_{3}\right)$. A function $x:[0, T] \rightarrow \mathbb{R}$ is a solution of the PBVP (1.1) on $[0, T]$ if and only if $x$ satisfies the integral equation

$$
\begin{aligned}
x(t)=e^{-P(t)}\left(D_{H K}\right) \int_{0}^{t} e^{P(s)}(f(s, x(s))+p(s) x(s)) d s \\
\quad+\frac{e^{-P(t)}}{e^{P(T)}-1}\left(D_{H K}\right) \int_{0}^{T} e^{P(s)}(f(s, x(s))+p(s) x(s)) d s
\end{aligned}
$$

for any $p(t) \in H K, p(t) \geq 0$ on $[0, T]$ with $P(t)=(H K) \int_{0}^{t} p(s) d s$ nonzero at $t=T$.

Proof. Let $p(t) \in H K, p(t) \geq 0$ on $[0, T]$ satisfy $P(T)=(H K) \int_{0}^{T} p(s) d s \neq 0$. Assume first that $x:[0, T] \rightarrow \mathbb{R}$ is a solution of PBVP (1.1). Then $x \in C([0, T])$. Hence, by Lemma 2.3, the distributional derivative $D x$ of $x$ exists and is $D_{H K}$-integrable. Applying (1.1), we obtain

$$
e^{P(t)}(D x+p(t) x)=e^{P(t)}(f(t, x)+p(t) x) .
$$

By Lemma 2.7 and integrating (3.2),

$$
x(t)=e^{-P(t)} x(0)+e^{-P(t)}\left(D_{H K}\right) \int_{0}^{t} e^{P(s)}(f(s, x(s))+p(s) x(s)) d s
$$

and

$$
x(0)=x(T)=\frac{1}{e^{P(T)}-1}\left(D_{H K}\right) \int_{0}^{T} e^{P(s)}(f(s, x(s))+p(s) x(s)) d s .
$$

Thus $x$ is a solution of the integral equation (3.1).

Conversely, assume that $x$ satisfies (3.1), or equivalently, the integral equation

$$
\begin{aligned}
e^{P(t)} x(t)=\left(D_{H K}\right) \int_{0}^{t} e^{P(s)}(f(s, x(s))+p(s) x(s)) d s \\
+\frac{1}{e^{P(T)}-1}\left(D_{H K}\right) \int_{0}^{T} e^{P(s)}(f(s, x(s))+p(s) x(s)) d s .
\end{aligned}
$$

Differentiation of (3.3) gives

$$
e^{P(t)}(D x+p(t) x)=e^{P(t)}(f(t, x)+p(t) x) \text { for all } t \in[0, T] .
$$

Hence, $x$ satisfies the differential equation (1.1) on $[0, T]$. Moreover, inserting $t=0$ and $t=T$ in (3.1) yields $x(0)=x(T)$. Thus $x$ is a solution of the PBVP (1.1). 
We now give the well-known Schauder fixed point theorem, which is used to prove the existence of solutions of the PBVP (1.1).

Lemma 3.2 ([13, Theorem 6.15], Schauder theorem). Let $M$ be a convex, closed subset of a normed vector space $X$. Let $\mathscr{A}$ be a continuous map of $M$ into a compact subset $K$ of $M$. Then $\mathscr{A}$ has a fixed point.

We are now ready to give our main results.

THEOREM 3.3. If the distribution $f$ in the PBVP (1.1) satisfies assumptions $\left(\mathrm{D}_{1}\right)-\left(\mathrm{D}_{3}\right)$, then there exists at least one solution of the PBVP (1.1).

Proof. Let

$$
g(t, x)=f(t, x)+p(t) x, \quad t \in[0, T], x \in[y, z],
$$

where $p(t) \in H K, p(t) \geq 0$ on $[0, T]$ and $P(T)=(H K) \int_{0}^{T} p(s) d s \neq 0$. It follows from $\left(\mathrm{D}_{1}\right)-\left(\mathrm{D}_{3}\right)$ that $g(\cdot, x(\cdot)) \in D_{H K}$ for all $x \in[y, z], g(t, \cdot)$ is continuous for all $t \in[0, T]$ and

$$
D y+p y \leq g(\cdot, x) \leq D z+p z, \quad \text { for each } x \in[y, z] .
$$

Define a mapping $\mathscr{A}$ on $[y, z]$ by

$$
\begin{aligned}
\mathscr{A} x(t)=e^{-P(t)}\left(D_{H K}\right) \int_{0}^{t} e^{P(s)} g(s, x(s)) d s & \\
& +\frac{e^{-P(t)}}{e^{P(T)}-1}\left(D_{H K}\right) \int_{0}^{T} e^{P(s)} g(s, x(s)) d s, \quad t \in[0, T] .
\end{aligned}
$$

It follows from (3.6) that, for each $x \in[y, z]$,

$$
\begin{aligned}
D(\mathscr{A} x) & =g(\cdot, x)-p \mathscr{A} x \quad \text { on }[0, T], \\
\mathscr{A} x(0) & =\mathscr{A} x(T) .
\end{aligned}
$$

Let $w=\mathscr{A} y-y$. Then, by $\left(\mathrm{D}_{1}\right),(3.4)$ and (3.7),

$$
D w+p w \geq 0, \quad w(0) \geq w(T),
$$

It follows from (3.8) that

$$
e^{P(t)} w(t) \geq w(0), \quad t \in[0, T] .
$$

Let $t=T$ in (3.9), and note that $w(0) \geq w(T)$. Then

$$
e^{P(T)} w(0) \geq e^{P(T)} w(T) \geq w(0),
$$

which implies that

$$
w(0) \geq 0 .
$$

In view of (3.9) and (3.10) we then have $w=\mathscr{A} y-y \geq 0$, that is, $y \leq \mathscr{A} y$. We can similarly verify that $\mathscr{A} z \leq z$. Hence (3.6) defines a mapping $\mathscr{A}:[y, z] \rightarrow[y, z]$. 
Since $y, z$ are continuous functions on $[0, T]$, they are also bounded on $[0, T]$. Thus, $\mathscr{A}([y, z])$ is uniformly bounded on $[0, T]$ for all $x \in[y, z]$.

Let $t_{1}, t_{2} \in[0, T]$. Then, by (3.6), for each $x \in[y, z]$,

$$
\begin{gathered}
\mathscr{A} x\left(t_{1}\right)-\mathscr{A} x\left(t_{2}\right)=e^{-P\left(t_{1}\right)}\left(D_{H K}\right) \int_{t_{2}}^{t_{1}} e^{P(s)} g(s, x(s)) d s \\
+\left(e^{P\left(t_{2}\right)-P\left(t_{1}\right)}-1\right) \mathscr{A} x\left(t_{2}\right) .
\end{gathered}
$$

Since $p(t) \in H K, p(t) \geq 0, P(t)=(H K) \int_{0}^{t} p(s) d s$ is continuous and so is uniformly continuous on $[0, T]$. Then, for all $\varepsilon>0$, there exists $\delta>0$ such that

$$
\left|e^{P\left(t_{2}\right)-P\left(t_{1}\right)}-1\right| \leq \varepsilon \quad \text { whenever } t_{1}, t_{2} \in[0, T] \text { and }\left|t_{2}-t_{1}\right| \leq \delta .
$$

It is easy to see that $e^{P(t)} \in C([0, T]) \cap \mathcal{B V}$ (and the same is true for $\left.e^{-P(t)}\right)$ on $[0, T]$. Hence, there exists $M>0$ such that

$$
\frac{1}{M}<e^{P(t)}<M, \quad t \in[0, T]
$$

The result $e^{P(t)} \in \mathcal{B V}$ on $[0, T]$ implies by Lemma 2.7 that $e^{P(t)} g(t, x(t))$ is $D_{H K^{-}}$ integrable on $[0, T]$, because $g(\cdot, x)$ is $D_{H K}$-integrable for all $x \in[y, z]$. This result and the monotonicity of $g(t, \cdot)$ for all $t \in[0, T]$ imply that

$$
\begin{aligned}
\left(D_{H K}\right) \int_{t_{2}}^{t_{1}} e^{P(s)} g(s, y(s)) d s & \leq\left(D_{H K}\right) \int_{t_{2}}^{t_{1}} e^{P(s)} g(s, x(s)) d s \\
& \leq\left(D_{H K}\right) \int_{t_{2}}^{t_{1}} e^{P(s)} g(s, z(s)) d s .
\end{aligned}
$$

On the other hand, the properties of $\mathscr{A}$ imply that

$$
y\left(t_{2}\right) \leq \mathscr{A} x\left(t_{2}\right) \leq z\left(t_{2}\right)
$$

Since $y, z \in C([0, T])$, there exists $N>0$ such that

$$
\begin{aligned}
\left|\mathscr{A} x\left(t_{1}\right)-\mathscr{A} x\left(t_{2}\right)\right| \leq M\left|\left(D_{H K}\right) \int_{t_{2}}^{t_{1}} e^{P(s)} g(s, x(s)) d s\right|+N \varepsilon \\
\leq M\left(\left|\left(D_{H K}\right) \int_{t_{2}}^{t_{1}} e^{P(s)} g(s, y(s)) d s\right|\right. \\
\left.+\left|\left(D_{H K}\right) \int_{t_{2}}^{t_{1}} e^{P(s)} g(s, z(s)) d s\right|\right)+N \varepsilon .
\end{aligned}
$$

Since $e^{P(t)} g(t, y(t))$ and $e^{P(t)} g(t, z(t))$ are $D_{H K}$-integrable on $[0, T]$, the primitives of $e^{P(t)} g(t, y(t))$ and $e^{P(t)} g(t, z(t))$ are continuous and so are uniformly continuous on $[0, T]$. Hence, by inequality $(3.12), \mathscr{A}([y, z])$ is equiuniformly continuous on $[0, T]$ for all $x \in[y, z]$. In view of the Ascoli-Arzelà theorem, $\mathscr{A}([y, z])$ is relatively compact. 
According to Lemma 3.2, we now only need to prove that $\mathscr{A}$ is continuous.

Let $x \in[y, z],\left\{x_{m}\right\}_{m \in \mathbb{N}}$ be a sequence in $[y, z]$ and $x_{m} \rightarrow x$ as $m \rightarrow \infty$. According to condition $\left(\mathrm{D}_{2}\right)$,

$$
f\left(\cdot, x_{m}\right) \rightarrow f(\cdot, x) \quad \text { as } m \rightarrow \infty
$$

Thus, by (3.4),

$$
e^{P(\cdot)} g\left(\cdot, x_{m}\right) \rightarrow e^{P(\cdot)} g(\cdot, x) \quad \text { as } m \rightarrow \infty .
$$

Hence, by Lemma 2.12 and (3.5),

$$
\lim _{m \rightarrow \infty} \int_{0}^{t} e^{P(s)} g\left(s, x_{m}(s)\right) d s=\int_{0}^{t} e^{P(s)} g(s, x(s)) d s, \quad t \in[0, T] .
$$

Therefore, $\lim _{m \rightarrow \infty} \mathscr{A} x_{m}(\cdot)=\mathscr{A} x(\cdot)$, which implies that $\mathscr{A}$ is continuous. Thus, $\mathscr{A}$ is a compact mapping. The result implies that $\mathscr{A}$ satisfies the hypotheses of Lemma 3.2, whence $\mathscr{A}$ has a fixed point, which is also the solution of PBVP (1.1).

We now consider the topological characterisation of the solution set of PBVP (1.1). First, we present the well-known Vidossich theorem.

Let $C_{u}(K, Y)$ be the space of all continuous mappings $x: K \rightarrow Y$, where $K$ is a compact convex subset of a normed space and $Y$ is a metric space equipped with the topology of uniform convergence. Denote by $B\left(t_{0}, \varepsilon\right)$ the closed ball with centre $t_{0}$ and radius $\varepsilon$. Denote by $\left.x\right|_{A}$ the restriction of the map $x$ to $A$.

Lemma 3.4 ([17, Corollary 1.2], Vidossich theorem). Let $K$ be a compact convex subset of a normed space, $Y$ a closed convex subset of a Banach space $Y_{0}, F$ a compact mapping $C_{u}(K, Y) \rightarrow C_{u}(K, Y)$. Suppose that there exist $t_{0} \in K$ and $y_{0} \in Y$ such that the following two conditions hold.

(i) $F(x)\left(t_{0}\right)=y_{0}(x \in C(K, Y))$.

(ii) For every $\varepsilon>0$,

$$
\left.x\right|_{K_{\varepsilon}}=\left.\left.y\right|_{K_{\varepsilon}} \Rightarrow F(x)\right|_{K_{\varepsilon}}=\left.F(y)\right|_{K_{\varepsilon}} \quad(x, y \in C(K, Y)),
$$

where $K_{\varepsilon}=B\left(t_{0}, \varepsilon\right) \cap K$.

Then the set of fixed points of $F$ is an $R_{\delta}$.

Recall that if a set is an $R_{\delta}$, it is homeomorphic to the intersection of a decreasing sequence of compact absolute retracts. Furthermore, Vidossich [17] pointed out that $R_{\delta}$ is a nonempty, compact and connected set.

We are now in a position to give the second main result.

THEOREM 3.5. Under the above assumptions $\left(\mathrm{D}_{1}\right)-\left(\mathrm{D}_{3}\right)$, the solution set $S_{1}$ of $P B V P(1.1)$ on $[0, T]$ is an $R_{\delta}$.

Proof. In Theorem 3.3, we have proved that the mapping $\mathscr{A}:[y, z] \rightarrow[y, z]$ is compact. According to the proof of Theorem 3.3, it is also easy to see that conditions (i) and (ii) of Lemma 3.4 hold. Thus, by Lemma 3.4, the solution set $S_{1}$ is an $R_{\delta}$. The proof is therefore complete. 
Corollary 3.6. Assume that $q(x)$ is continuous with respect to $x$, where $x \in C([0, T])$, and $\mu(t)$ is $D_{H K}$-integrable on $[0, T]$. Then the PBVP

$$
D x+q(x)=\mu(t), \quad x(0)=x(T),
$$

has at least one solution. Moreover, the solution set is an $R_{\delta}$.

Proof. Let

$$
f(t, x)=\mu(t)-q(x)
$$

and

$$
q_{+}=\|q(x)\|_{\infty} .
$$

Then, by the assumptions, it is easy to see that the distribution $f$ satisfies $\left(D_{1}\right),\left(D_{2}\right)$ and

$$
\mu-q_{+} \leq f(\cdot, x) \leq \mu+q_{+}, \quad \text { for all } x \in C([0, T]) .
$$

Let

$$
\begin{aligned}
y(t)=e^{-P(t)}\left(D_{H K}\right) \int_{0}^{t} e^{P(s)}\left(\mu(s)-q_{+}+p(s) x(s)\right) d s \\
\quad+\frac{e^{-P(t)}}{e^{P(T)}-1}\left(D_{H K}\right) \int_{0}^{T} e^{P(s)}\left(\mu(s)-q_{+}+p(s) x(s)\right) d s
\end{aligned}
$$

and

$$
\begin{aligned}
z(t)=e^{-P(t)}\left(D_{H K}\right) \int_{0}^{t} e^{P(s)}\left(\mu(s)+q_{+}+p(s) x(s)\right) d s & \\
& +\frac{e^{-P(t)}}{e^{P(T)}-1}\left(D_{H K}\right) \int_{0}^{T} e^{P(s)}\left(\mu(s)+q_{+}+p(s) x(s)\right) d s,
\end{aligned}
$$

where $p(t) \in H K, p(t) \geq 0$ on $[0, T]$, with $P(t)=(H K) \int_{0}^{t} p(s) d s$ nonzero at $t=T$. Thus, by Lemma 3.1 and Theorem 3.3, PBVP (1.1) has at least one solution, and by Theorem 3.5, the solution set is an $R_{\delta}$. The proof is therefore complete.

Remark 3.7. According to Corollary 3.6, Theorem 3.3 generalises [11, Theorem 7] and Theorem 3.5 extends [10, Theorem 5.1], because the $D_{H K}$-integral includes the Lebesgue integral and the distributional derivative contains the ordinary derivative.

We now give an example to illustrate the above results.

EXample 3.8. Consider the PBVP given by

$$
D x+\arctan x=D S, \quad x(0)=x(2),
$$

where $x \in C([0,2])$ and $D S$ is the distributional derivative of

$$
S(t)=\sum_{n=1}^{\infty} \frac{\sin 7^{n} \pi t}{2^{n}} .
$$

Then the PBVP (3.17) has at least one solution. Moreover, the solution set is an $R_{\delta}$. 
Proof. The PBVP (3.17) can be regarded as a PBVP of the form (3.13), where

$$
\mu=D S, \quad q(x)=\arctan x, \quad t \in[0,2], x \in C([0,2]) .
$$

It is easy to see that $\mu(t)$ and $q(x)$ satisfy the hypotheses of Corollary 3.6. Hence, the result holds.

It is well known that the Weierstrass function $S(t)$ in (3.18) is continuous but differentiable nowhere on $\mathbb{R}$ (for details, see [3]). Then, by Example 2.4, the distributional derivative $D S$ is neither $H K$-integrable nor Lebesgue integrable. Hence, [11, Theorem 7] and [10, Theorem 5.1] are not applicable in this case.

\section{References}

[1] D. D. Ang, K. Schmitt and L. K. Vy, 'A multidimensional analogue of the Denjoy-PerronHenstock-Kurzweil integral', Bull. Belg. Math. Soc. Simon Stevin 4 (1997), 355-371.

[2] T. S. Chew and P. Y. Lee, 'The topology of the space of Denjoy integrable functions', Bull. Aust. Math. Soc. 42 (1990), 517-524.

[3] G. H. Hardy, 'Weierstrass's non-differentiable function', Trans. Amer. Math. Soc. 17(3) (1916), 301-325.

[4] V. Lakshmikantham and S. Leela, 'Existence and monotone method for periodic solutions of firstorder differential equations', J. Math. Anal. Appl. 91 (1983), 237-243.

[5] V. Lakshmikantham and S. Leela, 'Remarks on first and second order periodic boundary value problems', Nonlinear Anal. 8 (1984), 281-287.

[6] P. Y. Lee, Lanzhou Lecture on Henstock Integration (World Scientific, Singapore, 1989).

[7] T. Y. Lee, 'A full descriptive definition of the Henstock-Kurzweil integral in the Euclidean space', Proc. London Math. Soc. 87 (2003), 677-700.

[8] Q. L. Liu and G. J. Ye, 'Some problems on the convergence of the distributional Denjoy integral', Acta Math. Sin. (Chin. Ser.) 54(4) (2011), 659-664 (in Chinese).

[9] Y. P. Lu, G. J. Ye, Y. Wang and W. Liu, 'The Darboux problem involving the distributional Henstock-Kurzweil integral', Proc. Edinb. Math. Soc. (2), in press.

[10] J. J. Nieto, 'Existence of periodic solutions for first-order differential equations', Appl. Math. Comput. 15 (1984), 221-232.

[11] J. J. Nieto, 'On the structure of the solution set for first-order differential equations', Appl. Math. Comput. 16 (1985), 177-187.

[12] W. Rudin, Real and Complex Analysis, 3rd edn (McGraw-Hill, New York, 1987).

[13] M. Schechter, An Introduction to Nonlinear Analysis (Cambridge University Press, Cambridge, 2004).

[14] Š. Schwabik and G. J. Ye, Topics in Banach Space Integration (World Scientific, Singapore, 2005).

[15] E. Talvila, 'The distributional Denjoy integral', Real Anal. Exchange 33 (2008), 51-82.

[16] C. C. Tisdell, 'Existence of solutions to first-order periodic boundary value problems', J. Math. Anal. Appl. 323 (2006), 1325-1332.

[17] G. Vidossich, 'A fixed-point theorem for funtion spaces', J. Math. Anal. Appl. 36 (1971), 581-587.

\section{WEI LIU, Department of Mathematics, Hohai University, Nanjing 210098, PR China e-mail: liuw626@hhu.edu.cn}


GUOJU YE, Department of Mathematics, Hohai University, Nanjing 210098, PR China

e-mail: yegj@hhu.edu.cn

YING WANG, Department of Mathematics, Hohai University,

Nanjing 210098, PR China

XUEYUAN ZHOU, Department of Mathematics, Hohai University, Nanjing 210098, PR China 